\title{
CKD-MBD: un caso di devastanti complicanze vertebrali
}

\author{
V. Martina ${ }^{1 *}$, M.A. Rizzo ${ }^{1 *}$, C. Uggetti ${ }^{2}$, L. Gravellone ${ }^{1}$, A. Giordano ${ }^{1}$, M. Egidi $^{3}$, \\ M. Gallieni ${ }^{1}$
}

${ }^{1}$ U.O.C. Nefrologia e Dialisi, Ospedale San Carlo Borromeo, Milano

${ }^{2}$ U.O.S. Neuroradiologia, Ospedale San Carlo Borromeo, Milano

${ }^{3}$ U.O.C. Neurochirurgia, Ospedale San Carlo Borromeo, Milano

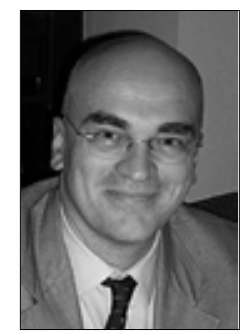

Maurizio Gallieni

Descriviamo il decorso delle alterazioni del metabolismo minerale e osseo nella lunga storia dialitica di una paziente di 80 anni affetta da insufficienza renale cronica avanzata secondaria a rene policistico, in trattamento emodialitico dal 1980. La patologia ossea esordiva con un quadro di iperparatiroidismo secondario, confermata da una biopsia ossea, trattata chirurgicamente nel 1993 con paratiroidectomia totale e autoinnesto in avambraccio destro. L'innesto risultava però non funzionante, determinando una malattia adinamica dell'osso associata a valori di paratormone inferiori a $10 \mathrm{ng} / \mathrm{L}$, calcemia e fosforemia ai limiti inferiori di norma e fisiologici livelli di fosfatasi alcalina. Nel 1997 e nel 2000 eseguiva rispettivamente discectomia L5-S1 e laminectomia decompressiva L3-S1, nel 2007 un posizionamento di protesi monocompartimentale mediale mininvasiva del ginocchio destro. Indagini radiologiche dimostravano la presenza di calcificazioni vascolari diffuse (bacino, ginocchio) in assenza di lesioni trofico-ischemiche. Per contrastare la tendenza all'ipocalcemia si introduceva terapia con calcitriolo orale.

A giugno 2009 esordiva una sintomatologia dolorosa agli arti inferiori bilateralmente; una TAC lombo-sacrale dimostrava importante listesi a livello L4-L5 e discopatia associata, anche a livello L5-S1, con compressione delle radici nervose; la valutazione neurochirurgica sconsigliava un trattamento chirurgico e poneva indicazione alla sola terapia antalgica che veniva inizialmente instau-

\footnotetext{
* Questi autori hanno contribuito nello stesso modo alla stesura del testo
}

rata con analgesici oppioidi, in particolare fentanil citrato, sostituito successivamente con buprenorfina, per l'insorgenza di vertigini. Si assisteva a un miglioramento della sintomatologia dolorosa ma anche alla progressiva comparsa di ipomobilità agli arti inferiori con grave compromissione della deambulazione, e ipostenia agli arti superiori.

Eseguiva, quindi, RMN encefalo e midollo spinale completata da TC, che dimostravano discopatie protrusive multiple, la più importante delle quali a livello C4-C5 con caratteristiche di ernia molle, determinante significativa riduzione del canale vertebrale e compressione midollare (Fig. 1A e Fig. 1B).

Collateralmente, si rilevavano, oltre a una riduzione diffusa del tenore calcico, caratteristiche cavità geodiche, espressione di una possibile presenza di amiloidosi dialitica da beta-2-microglobulina (Fig. 1C). La paziente veniva sottoposta ad intervento di discectomia cervicale C4-C5 con approccio anteriore più artrodesi cervicale con posizionamento di cage in titanio; la valutazione radiologica post-operatoria mostrava un adeguato posizionamento della cage con allineamento metamerico vertebrale conservato (Fig. 2).

La successiva riabilitazione permetteva un recupero della forza muscolare, soprattutto degli arti inferiori con parziale autonomia nei trasferimenti. Permaneva un deficit motorio dell'arto superiore destro. Nelle settimane seguenti, si assisteva tuttavia alla ricomparsa della sintomatologia clinica che progressivamente esitava nella recidiva della tetraparesi iperflessica in associazione a importante componente dolorosa. L'indagine TAC cervicale mostrava una regolare evoluzione artrodesica al livello trattato (C4-C5) ma la comparsa di una retrolistesi di C5 su C6 con erniazione del disco intersomatico corrispondente (Fig. 3). Pertanto, l'efficace intervento 


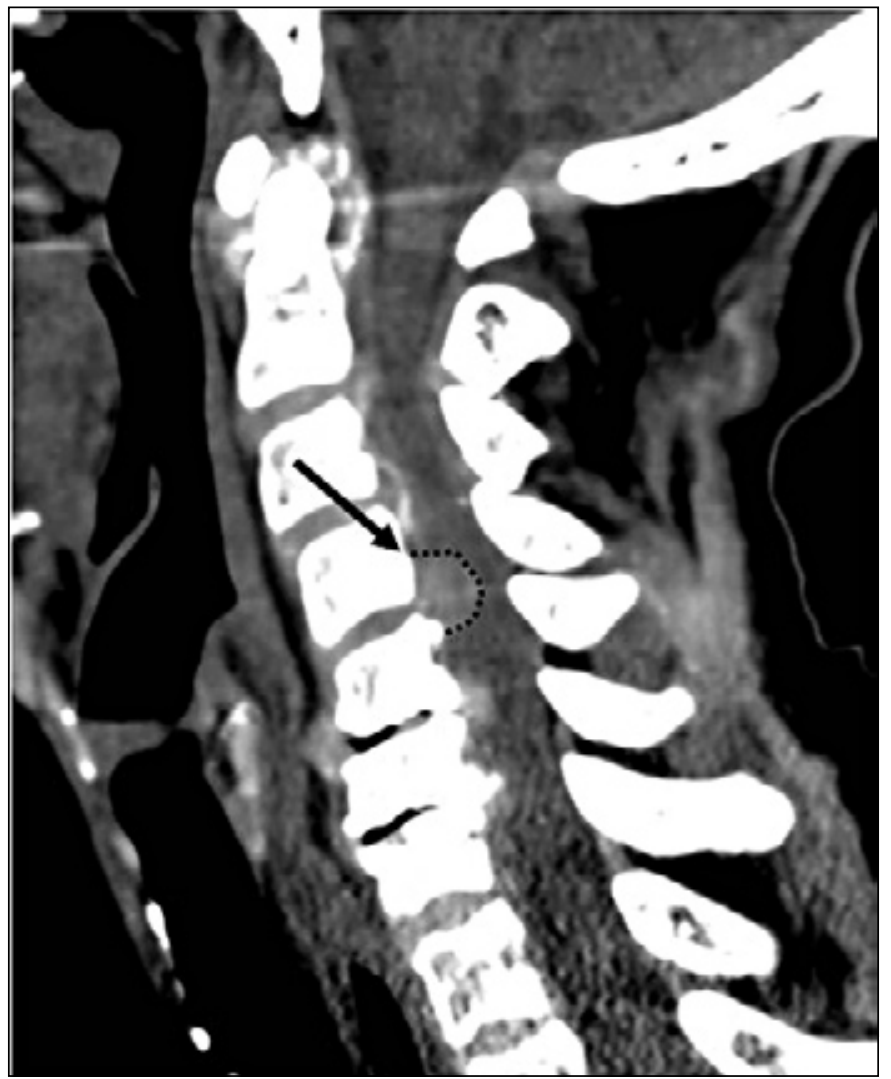

decompressivo e artrodesico al livello C4-C5 veniva vanificato dall'insorgenza di una instabilità giunzionale al livello caudale. La valutazione neurochirurgica sconsigliava ulteriori provvedimenti chirurgici.

Nelle settimane successive la sintomatologia clinica peggiorava e il quadro si complicava in tetraplegia, con insorgenza di piaghe da decubito e di exitus per insufficienza respiratoria.

\section{Discussione}

Le alterazioni del metabolismo osseo sono frequenti nel paziente con insufficienza renale cronica con notevole impatto sulla morbilità e mortalità (1-3). La complessa patologia ossea dei pazienti affetti da insufficienza renale cronica è oggi definita con il termine di Chronic Kidney Disease-Mineral and Bone Disorders (CKD-MBD), che comprende sia patologie ad elevato turnover, come quella conseguente a iperparatiroidismo secondario, sia patologie a basso turnover, come la malattia ossea adinamica $(4,5)$.

La patologia ossea ad elevato turnover è determinata da aumentata secrezione di PTH e caratterizzata istologicamente da eccessivo riassorbimento osseo (osteite fibrosa e osteopatia mista); la patologia ossea a basso turnover, invece, è determinata da un deficit assoluto o
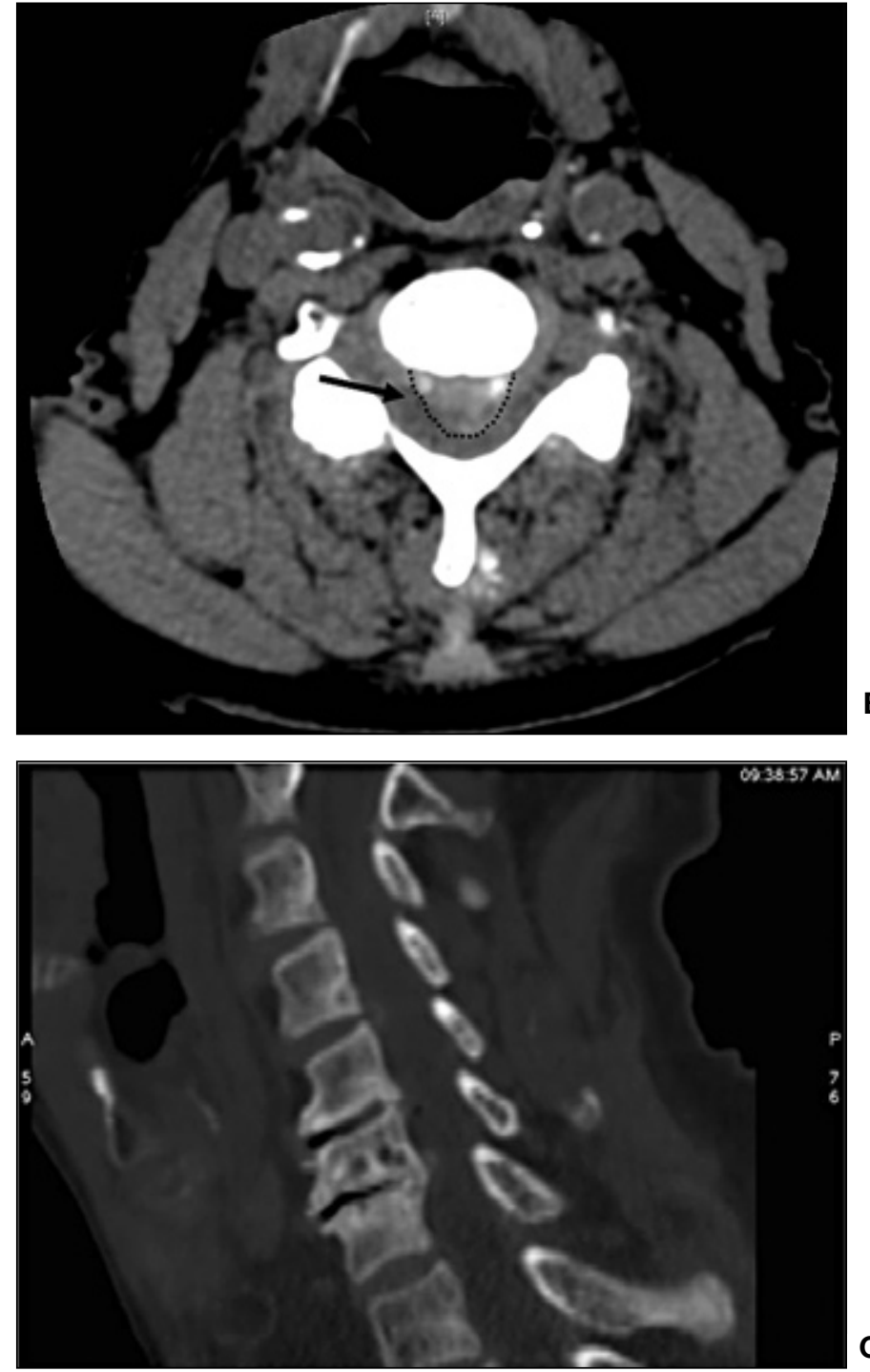

Fig. 1 - A) TAC rachide cervicale, sezione sagittale-mediana, pre-intervento. Si dimostrano erniazioni multiple dei dischi intersomatici, di cui la più voluminosa in sede C4-C5 con caratteristiche di ernia molle.

B) TAC rachide cervicale, sezione assiale, pre-intervento. Voluminosa erniazione posteriore mediana del disco C4-C5 (freccia, linea tratteggiata), che scolla il legamento longitudinale posteriore e determina una riduzione del diametro sagittale del canale rachideo di circa due terzi. C) TAC rachide cervicale, sezione sagittale-mediana, preintervento. Riduzione del tenore calcico di tutti i distretti, associato alla presenza di cavità geodiche vertebrali.

relativo di PTH e caratterizzata da assenza di processi di rimodellamento osseo (osso adinamico).

Nella patogenesi dell'iperparatiroidismo secondario, oltre al ruolo della ritenzione di fosfati, deficit di calcitriolo e ipocalcemia, è oggi discussa anche l'azione dell'ormone FGF23 (fibroblast growth factor 23): si tratta di un 


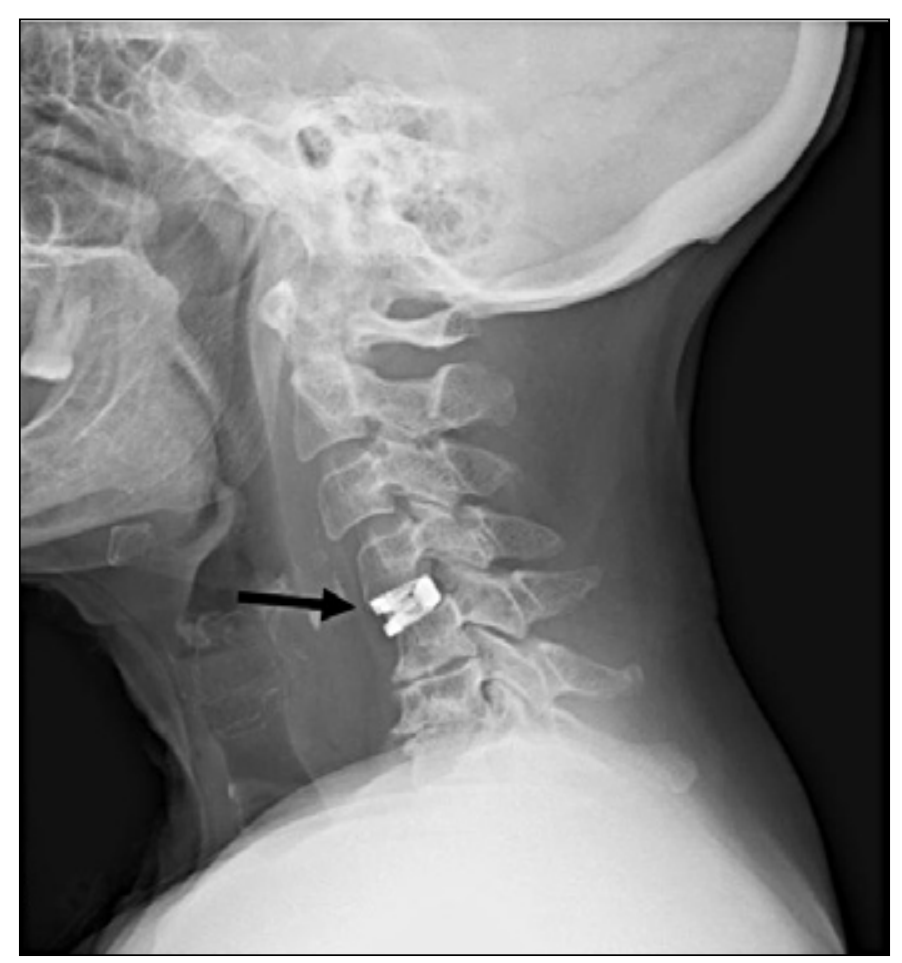

Fig. 2 - Radiografia del rachide cervicale in proiezione latere-laterale, immediato post-intervento. Un distanziatore intersomatico (cage in titanio, freccia) è adeguatamente posizionato, con conservato allineamento dei metameri vertebrali.

ormone che oltre ad inibire il riassorbimento tubulare renale di fosforo (un'azione fosfaturica, che contrasta l'iperparatiroidismo) riduce però anche la sintesi renale di 1-25D, un effetto che può indurre aumento del PTH $(6,7)$. L'iperparatiroidismo secondario si manifesta clinicamente con dolori ossei e maggior suscettibilità alle fratture; a ciò si aggiunge la tossicità sistemica del PTH sull'apparato cardiovascolare e la tendenza alle calcificazioni vascolari $(8,9)$. Il trattamento dell'iperparatiroidismo secondario si giova attualmente di diverse strategie terapeutiche basate innanzitutto sul controllo delliperfosforemia, sull'utilizzo di vitamina $\mathrm{D}$, in particolare degli attivatori selettivi del VDR, e dei calciomimetici in monoterapia $o$ in terapia combinata (10-13). La recente introduzione di cinacalcet ha permesso di ridurre le indicazioni alla paratiroidectomia totale, importante causa di evoluzione verso l'osso adinamico $(12,13)$, come è successo nel caso qui descritto.

L'Osso Adinamico (Adynamic Bone Disease-ADB) è predominante in dialisi peritoneale $(61 \%)$, ma ha una prevalenza significativa anche nei pazienti emodializzati (21\%). Le cause più frequenti sono la paratiroidectomia totale, l'età avanzata, l'uso di corticosteroidi, il diabete

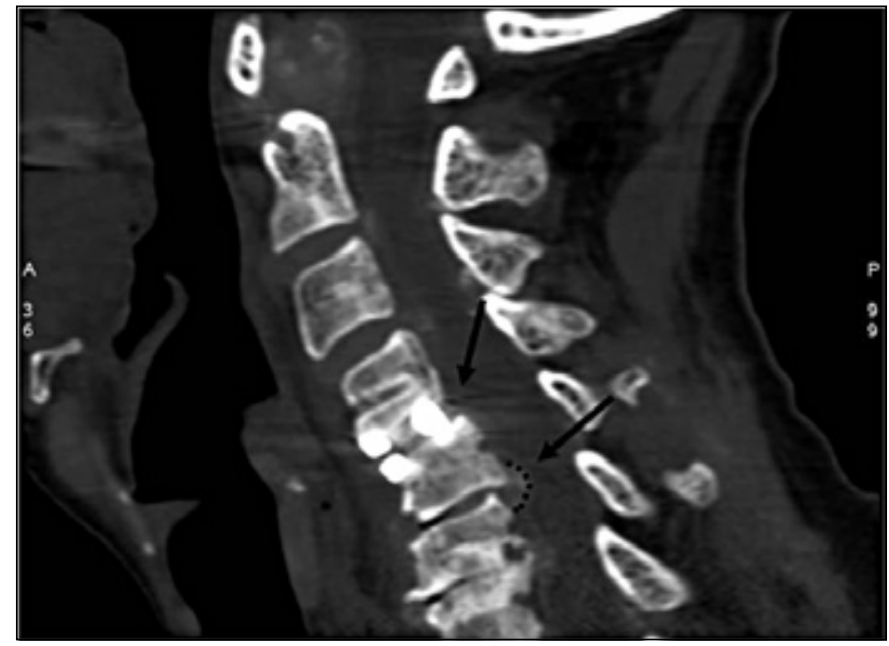

Fig. 3 - TAC rachide cervicale, sezione sagittale-mediana, 2 mesi post-intervento. Curva di cifosi con fulcro al passaggio C5-C6; retrolistesi di C5 su C6 con erniazione del disco inter-somatico corrispondente.

mellito, il trattamento con dosi eccessive di vitamina $\mathrm{D}$ e integratori di calcio, la dialisi peritoneale. La paratiroidectomia totale è causa di malattia adinamica dell'osso per la mancata azione del PTH sul turnover osseo. La patologia può rimanere clinicamente silente, ma è frequente l'associazione con dolori ossei, microfratture e ipercalcemia. Clinicamente si manifesta con debolezza muscolare prossimale, dolore localizzato a livello vertebrale, fratture costali, vertebrali e del bacino. Si associa in modo marcato alla presenza di calcificazioni vascolari ed extra-scheletriche: il basso turnover scheletrico ridurrebbe la capacità del tessuto osseo di tamponare le escursioni calcio-fosforiche con accumulo di calcio e fosforo nei tessuti extra-scheletrici, soprattutto nel contesto della parete vasale. La sintomatologia clinica può essere confermata dalla biopsia ossea; dal punto di vista bioumorale, si riscontrano valori di PTH fortemente depressi e fosfatasi alcalina non elevata. L'approccio terapeutico è quello di ridurre l'apporto di calcio e vitamina $D$ ristabilendo (se è presente una quantità anche modesta di tessuto paratiroideo) l'attività del PTH $(14,15)$.

La storia della paziente è esemplificativa delle due estreme complicanze della CKD-MBD, dimostrando come una forma patologica possa evolvere nell'altra a causa del trattamento terapeutico stesso. Risalendo l'esordio della sua malattia ai decenni passati, la paratiroidectomia con autoinnesto era un tipico approccio terapeutico per trattare l'iperparatiroidismo grave. Tale procedura, in caso di mancato funzionamento dell'autoinnesto, comportava l'insorgenza di ipoparatiroidismo. Il nostro caso clinico dimostra come le conseguenze dell'osso adinamico non 
siano meno invalidanti di quelle che insorgono in corso di iperparatiroidismo secondario e che spesso richiedono interventi invasivi in stadi avanzati e generalizzati di danno osseo con una difficile riuscita a lungo termine. E significativo segnalare come in questa paziente, che ha sempre mantenuto un buon controllo clinico e nutrizionale, le ripercussioni neurologiche delle complicanze ossee siano state più rilevanti di quelle vascolari, rappresentate da calcificazioni non associate a lesioni trofiche o ad eventi cardiaci o cerebrovascolari. Inoltre non è possibile escludere una patologia ossea da deposito di beta-2-microglobulina, vista la lunga storia dialitica della paziente e la presenza radiologica di cisti o geodi ossei in sede vertebrale, descritte in letteratura come aspetti radiologici tipici dell'amiloidosi da dialisi (16).

Ad oggi è attivo il dibattito sulle indicazioni alla paratiroidectomia proprio alla luce delle possibili conseguenze dell'intervento e dei nuovi approcci farmacologici, quali i calciomimetici. Nessun livello di PTH esclude la possibilità di un tentativo di terapia medica; la paratiroidectomia è indicata in presenza di calcificazioni extrascheletriche, arteriolopatia uremica calcifica, sintomatologia clinica $(3,17)$. D'altra parte l'approccio chirurgico sembra avere un miglior profilo costo-efficacia, anche se la mortalità post-operatoria non è trascurabile (18).

Alcuni dati della letteratura dimostrano pari efficacia nel controllo dei valori di PTH di una paratiroidectomia senza autotrapianto rispetto alla modalità subtotale $\mathrm{e}$ con autotrapianto: Lorenz et al (19) hanno confrontato il decorso post-operatorio di 23 pazienti sottoposti a paratiroidectomia senza autoinnesto con 64 pazienti sottoposti a paratiroidectomia con autoinnesto; questi autori, con un follow-up medio di 27 mesi, riscontrano una pari efficacia dei due trattamenti chirurgici e ipotizzano l'esistenza di nidi cellulari isolati in grado di proliferare e concludono che la paratiroidectomia totale permette una migliore prevenzione delle recidive. Propongono, per ridurre le complicanze da ipoparatiroidismo post-chirurgico, di eseguire una criopreservazione di tessuto paratiroideo per un eventuale successivo autoinnesto (19).

Recentemente, è stato descritto un caso di ipoparatiroidismo in paziente sottoposto a trapianto di rene, complicato da severe alterazioni ossee, trattato con PTH ricombinante (Teriparatide) (20). Attualmente non vengono descritti in letteratura casi di pazienti in emodialisi affetti da ipoparatiroidismo iatrogeno trattati con teriparatide, ma alla luce delle gravi complicanze ossee, come quelle descritte nel nostro caso clinico, tale terapia potrebbe costituire un’opportunità di trattamento.

\section{Riassunto}

La complessa patologia ossea dei pazienti affetti da insufficienza renale cronica è oggi definita Chronic Kidney Disease-Mineral and Bone Disorders (CKD-MBD) e comprende quadri patologici differenti tra i quali l'osso adinamico (Adynamic Bone Disease-ABD). Le conseguenze dell'ABD non sono meno invalidanti di quelle che insorgono in corso di iperparatiroidismo secondario. Talvolta le manifestazioni cliniche di ABD, come le complicanze vertebrali a lungo temine qui descritte, possono avere ripercussioni extrascheletriche tali da richiedere necessariamente un approccio terapeutico neurochirurgico invasivo, ma l'esito negativo dell'intervento effettuato sulla nostra paziente, per l'insorgenza di un'instabilità secondaria, sottolinea la difficoltà di successo quando si opera selettivamente in una situazione clinica di globale deterioramento del tessuto osseo. A questo proposito potrebbe essere valorizzato l'utilizzo di tecniche chirurgiche meno rigide dell'artrodesi strumentata per compensare la minore elasticità e resistenza dell'osso. Da ciò si desume l'importanza di un attento followup clinico del paziente e della necessità di una fattiva collaborazione con altri specialisti (neurologo, neurochirurgo, radiologo) per la prevenzione delle complicanze a lungo termine della patologia ossea del paziente dializzato.

Parole chiave: Dialisi, Iperparatiroidismo, Osso adinamico, Paratiroidectomia, Amiloidosi, ß2-microglobulina

\author{
Indirizzo degli Autori: \\ Maurizio Gallieni, MD \\ U.O. Nefrologia e Dialisi \\ Ospedale San Carlo Borromeo \\ Via Pio Secondo 3 \\ 20153 Milano \\ gallieni.maurizio@sancarlo.mi.it
}




\section{TEST di VERIFICA - 2}
1) La definizione “CKD-MBD” comprende?
a) Patologia ossea ad alto turnover
b) Patologia ossea a basso turnover
c) Entrambe
d) Nessuna

\section{2) Che cosa si intende per "Osso Adinamico"?}

a) Assenza di processi di rimodellamento osseo

b) Malattia ossea ad elevato turnover

c) Eccessivo riassorbimento osseo

d) Non si tratta di una patologia ossea

\section{3) Qual è il ruolo di FGF23?}

a) E un ormone fosfaturico con effetto ulteriore di inibizione della sintesi di $1-25(\mathrm{OH})_{2}$ vitD

b) Aumenta la fosforemia, assieme al PTH

c) Favorisce l'attivazione della vitamina D

d) È una forma inattiva di PTH

Le risposte corrette alle domande sono a pag. 11

\section{Bibliografia}

1. Block GA, Hulbert-Shearon TE, Levin NW, Port FK. Association of serum phosphorus and calcium $\mathrm{x}$ phosphate product with mortality risk in chronic hemodialysis patients: a national study. Am J Kidney Dis 1998; 31: 607-17.

2. Block GA, Klassen PS, Lazarus JM, Ofsthun N, Lowrie EG, Chertow GM. Mineral metabolism, mortality, and morbidity in maintenance hemodialysis. J Am Soc Nephrol 2004; 15: 2208-18.

3. Blacher J, Guerin AP, Pannier B, Marchais SJ, London GM. Arterial calcifications, arterial stiffness, and cardiovascular risk in end-stage renal disease. Hypertension 2001; 38: 938-42.

4. Messa $\mathrm{P}$ et al.; Società italiana di Nefrologia. Linee Guida SIN Osteodistrofia Renale G Ital Nefrol. 2003; 20 (Suppl 24): S83-95. http://www.sin-italy.org/pdf/linee_guida/2003_LG_OD.pdf

5. KDIGO Clinical practice guideline for the diagnosis, evaluation, prevention, and treatment of chronic kidney disease-mineral and bone disorder (CKD-MBD). Kidney Int 2009; 76(Suppl 113): S1-130.

6. Quarles LD. Endocrine functions of bone in mineral metabolism regulation. J Clin Invest 2008; 118: 3820-28.
7. Gutiérrez OM, Mannstadt M, Isakova T, et al. Fibloblast Growth Factor 23 and Mortality among Patients Undergoing Hemodialysis. New Engl J Med 2008; 359: 584-92.

8. Malberti F. Fisopatologia dell'iperparatiroidismo secondario. Giornale di Tecniche Nefrologiche \& Dialitiche 2009; 1 : 30-6.

9. Mazzaferro S, Pasquali M. Fisiopatologia del metabolismo calcio fosforo. patogenesi delliperparatiroidismo: nuove acquisizioni. Giornale di Tecniche Nefrologiche \& Dialitiche 2007; 3/4: 3-7.

10. Gallieni M, Pasho S, Crovetto C, Missaglia E. Il trattamento dell'iperparatiroidismo secondario con gli analoghi della vitamina D. Giornale di Tecniche Nefrologiche \& Dialitiche 2007; 3/4: 21-7.

11. Bandini S. Osteodistrofia renale: prevenzione e trattamento. Giornale di Tecniche Nefrologiche \& Dialitiche 2006; 1: 6-13.

12. Messa P. Uso dei calciomimetici nell'iperparatiroidismo secondario. Giornale di Tecniche Nefrologiche \& Dialitiche 2009; 1 : 43-50.

13. Locatelli F, Pontoniero G., Limardo M. I calciomimetici nell'iperparatiroidismo primitivo e secondario. Giornale di Tecniche Nefrologiche \& Dialitiche 2009; 1: 3-7.

14. Bandini S. Osteodistrofia Renale: Aspetti Patogenetici. Giornale di Tecniche Nefrologiche \& Dialitiche 2006; 1 : 1-5.

15. Frazao JM, Martins P. Adynamic bone disease: clinical and therapeutic implications. Curr Opin Nephrol Hypertension 2009; 18: 303-07.

16. Brancaccio D, Gallieni M. Patologia da accumulo di beta2-microglobulina. Trattato Italiano di Dialisi, 1999; 8.2: $777-85$.

17. Malberti F. Il trattamento chirurgico dell'iperparatiroidismo nel paziente uremico. Ruolo attuale della paratiroidectomia: aspetti epidemiologici e nuove acquisizioni. Giornale di Tecniche Nefrologiche \& Dialitiche 2007; 3/4: 41-7.

18. Narayan R, Perkins RM, Berbano EP, et al. Parathyroidectomy versus cinacalcet hydrochloride-based medical therapy in the management of hyperparathyroidism in ESRD: a cost utility analysis. Am J Kidney Dis 2007; 49: 801-13.

19. Lorenz K, Ukkat J, Sekulla C, Gimm O, Brauckhoff M, Dralle H. Total parathyroidectomy without autotransplantation for renal hyperparathyroidism: experience with a qPTH-controlled protocol. World J Surg 2006; 30: 743-51.

20. Mahajan A, Narayanan M, Jaffers G, Concepcion L. Hypoparathyroidism associated with severe mineral bone disease postrenal transplantation, treated successfully with recombinant PTH. Hemodial Int 2009; 13: 547-50. 